\title{
Influence of partial replacement of olive oil on frying performance of palm olein.
}

\begin{abstract}
The influence of partial replacement of palm olein (POo) with olive oil (Oo), (25 and 50\%, $\mathrm{w} / \mathrm{w})$ was investigated during five consecutive days of frying. The results indicated that frying performance of POo was significantly $(\mathrm{P}<0.05)$ influenced by partial replacement with olive oil. The highest change in peroxide value (PV), anisidine value (AV), totox value (TV), total polar compound (TPC), viscosity and melting point was shown by the control sample; whereas the replacement of $50 \%(\mathrm{w} / \mathrm{w})$ palm olein with $50 \%(\mathrm{w} / \mathrm{w})$ olive oil exhibited the least changes in PV, AV, TV, TPC, viscosity and melting point during the frying process. This study suggests that the partial replacement of palm olein containing a high proportion of saturated fatty acids (i.e., palmitic acid) with olive oil containing a high content of monounsaturated fatty acid (i.e., oleic acid) can provide oil blends with higher chemical stability against oxidation. On the other hand, the prepared oil blend remained liquid at ambient temperature, thereby enhancing the physical stability induced by partial replacement with olive oil.
\end{abstract}

Keyword: Partial replacement; Palm olein; Olive oil. 